\section{Taking the hydro out of hydrofracturing: Application of ultra-light weight proppants to cryogenic liquid nitrogen as a fracturing fluid}

Élimination de I'hydroélectricité par hydrofracturation : application d'agents de soutènement ultra-légers à l'azote liquide cryogénique comme fluide de fracturation
Amna Ahmed, Amna Majeed and Teresa Zhu

\begin{abstract}
In the last decade, hydraulic fracturing has rapidly gained popularity worldwide, emerging as the leading method of natural gas extraction in the United States. However, the practice remains controversial due to its contribution to greenhouse gas emissions and the contamination of freshwater used in fracturing fluids. Although waterless fracturing fluids have been developed, including those using $\mathrm{N}_{2}, \mathrm{CO}_{2}$, oil, and alcohol, their application has been limited largely due to reduced fracturing power. Recent research has demonstrated that cryogenic nitrogen may prove a viable alternative, if this issue is properly addressed. Addition of durable, lightweight proppants is one way to increase fracturing power. This study aims to investigate the effect of proppant addition on the fracturing capabilities of cryogenic nitrogen. Three ultra-lightweight proppants will be combined with liquid nitrogen and fracturing power will be measured using triaxial stress tests. This novel approach has not yet been explored and will open more avenues of research into sustainable and efficient fracturing using cryogenic nitrogen.
\end{abstract}

\section{Résumé}

$\mathrm{Au}$ cours de la dernière décennie, la fracturation hydraulique a rapidement gagné en popularité dans le monde entier, devenant la principale méthode d'extraction du gaz naturel aux États-Unis. Cependant, cette pratique reste controversée en raison de sa contribution aux émissions de gaz à effet de serre et à la contamination de l'eau douce utilisée dans les fluides de fracturation. Bien que des fluides de fracturation sans eau aient été mis au point, y compris ceux utilisant du $\mathrm{N}_{2}$, du $\mathrm{CO}_{2}$, de l'huile et de l'alcool, leur application a été limitée en grande partie en raison de la réduction du pouvoir de fracturation. Des études récentes ont démontré que l'azote cryogénique peut s'avérer une alternative viable, si cette question est correctement traitée. L'ajout d'agents de soutènement durables et légers est un moyen d'augmenter le pouvoir de fracturation. Cette étude a pour but d'étudier l'effet de l'ajout d'un agent de soutènement sur les capacités de fracturation de l'azote cryogénique. Trois agents de soutènement ultra-légers seront combinés avec de l'azote liquide et le pouvoir de fracturation sera mesuré à l'aide de tests de résistance triaxiaux. Cette approche novatrice n'a pas encore été explorée et ouvrira davantage de pistes de recherche sur la fracturation durable et efficace à l'aide d'azote cryogénique.

\footnotetext{
*University of Toronto St. George, Toronto, Ontario, Canada

Université de Toronto St. George, Toronto, Ontario, Canada

${ }^{\dagger}$ Lower division / Division inférieure

$\ddagger$ 3rd place / 3ème place
} 